\title{
Metarel: An Ontology to Support the Inferencing of Semantic Web
}

Relations within Biomedical Ontologies

\section{Ward Blondé}

Dept. of Applied Math., Biometrics and Process Control

University of Ghent, Belgium

ward.blonde@ugent.be

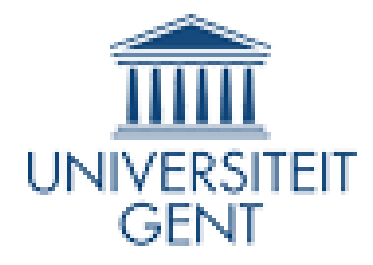




\section{Contents}

1. Introduction

2. Approach and architecture

3. Relational closures

4. Results

5. Conclusions 


\section{Introduction}

- The ever increasing biological knowledge needs automated integration

- Ontologies provide a good scaffold for such integration:

- High expressivity for detailed knowledge

- Extensional knowledge can be inferred from a maintained core (the intensional knowledge)

$\rightarrow$ The Semantic Web can help us out 


\section{Introduction}

- Biomedical ontologies contain only the intensional knowledge $\rightarrow$ the scaffold

- The extensional knowledge can be inferred from this scaffold by computational reasoning

- The rules that say which inferences can be made are mostly provided by the semantics of the relations in the ontology

$\rightarrow$ We automated inferences starting from Metarel, an ontology that captures the semantics of relations 


\section{Next section}

1. Introduction

2. Approach and architecture

3. Relational closures

4. Results

5. Conclusions 


\section{Reasoning with METAREL}

- Metarel was created to allow relational inferencing in ontologies

- It is a meta-ontology for relations that exists in OBOF and RDF, compatible with RO

- Relational closures like reflexivity, transitivity, compositions, etc., can be created by using SPARUL, the update language for RDF

- An application in the RDF store BioGateway on the Gene Ontology (GO) and the GO Annotations (GOA) created 200 million inferences 


\section{BioGateway}

3P SPARQL - Mozilla Firefox

Eile Edit View History Bookmarks Iools Help

(C) - C $\mathrm{C}$ \& $\mathrm{C}$ http://www.semantic-systems-biology.org/biogateway/querying

G. Google

Do Most Visited

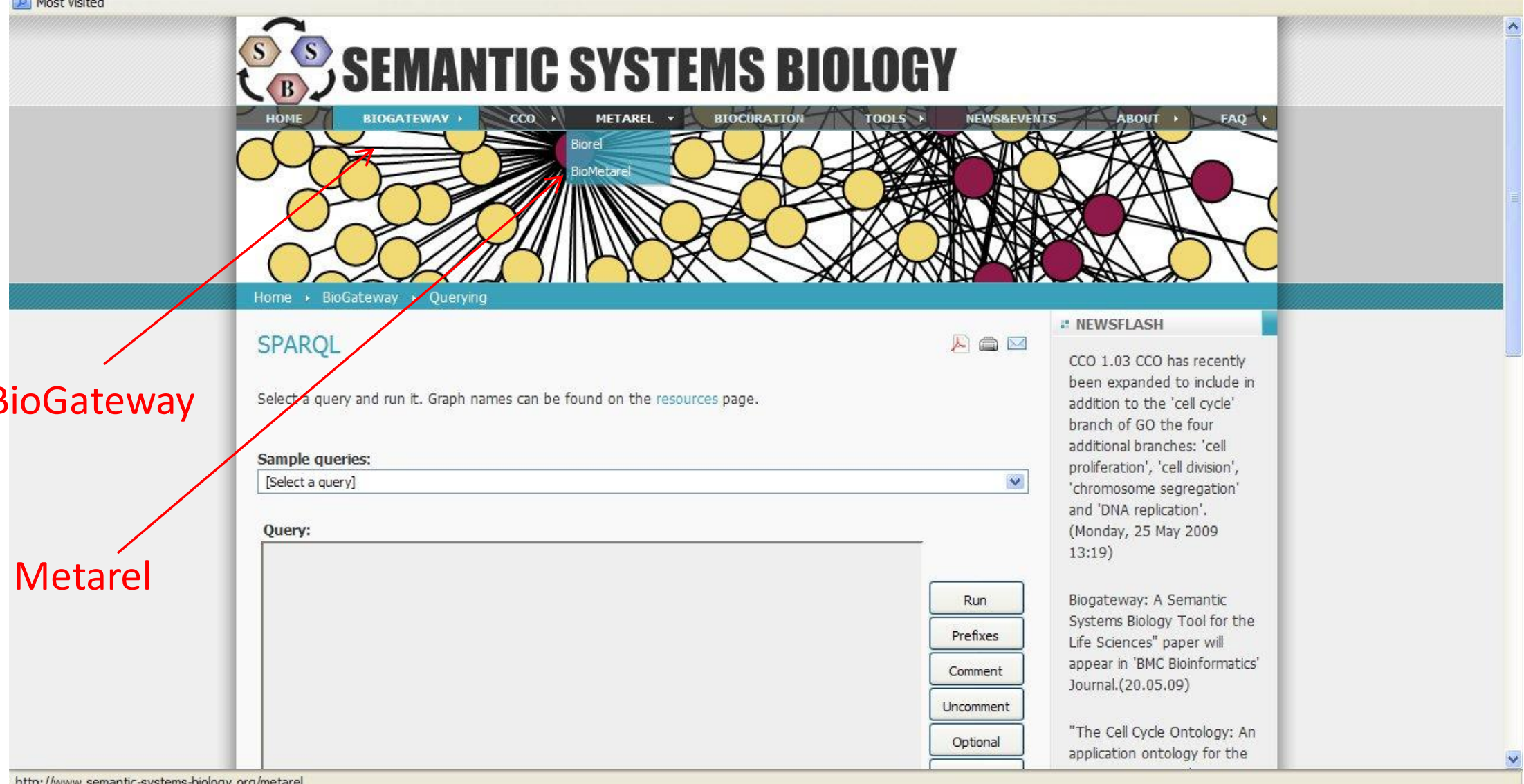

http://www.semantic-systems-biology.org/metarel

Erick Antezana, Ward Blondé, Mikel Egana, Alistair Rutherford, Robert Stevens, Bernard De Baets, Vladimir Mironov and Martin Kuiper. BioGateway: a Semantic Systems Biology tool for the life sciences, BMC, 2009 


\section{Metarel architecture}

Two hierarchies for relations:

1. Is related to: Classes in this hierarchy can be written with a verb in the third person singular, like 'is a', 'is part of', 'is located in', ...

2. Relation type: Classes in this hierarchy are meta-classes. Classes in hierarchy 1 are instances in this hierarchy.

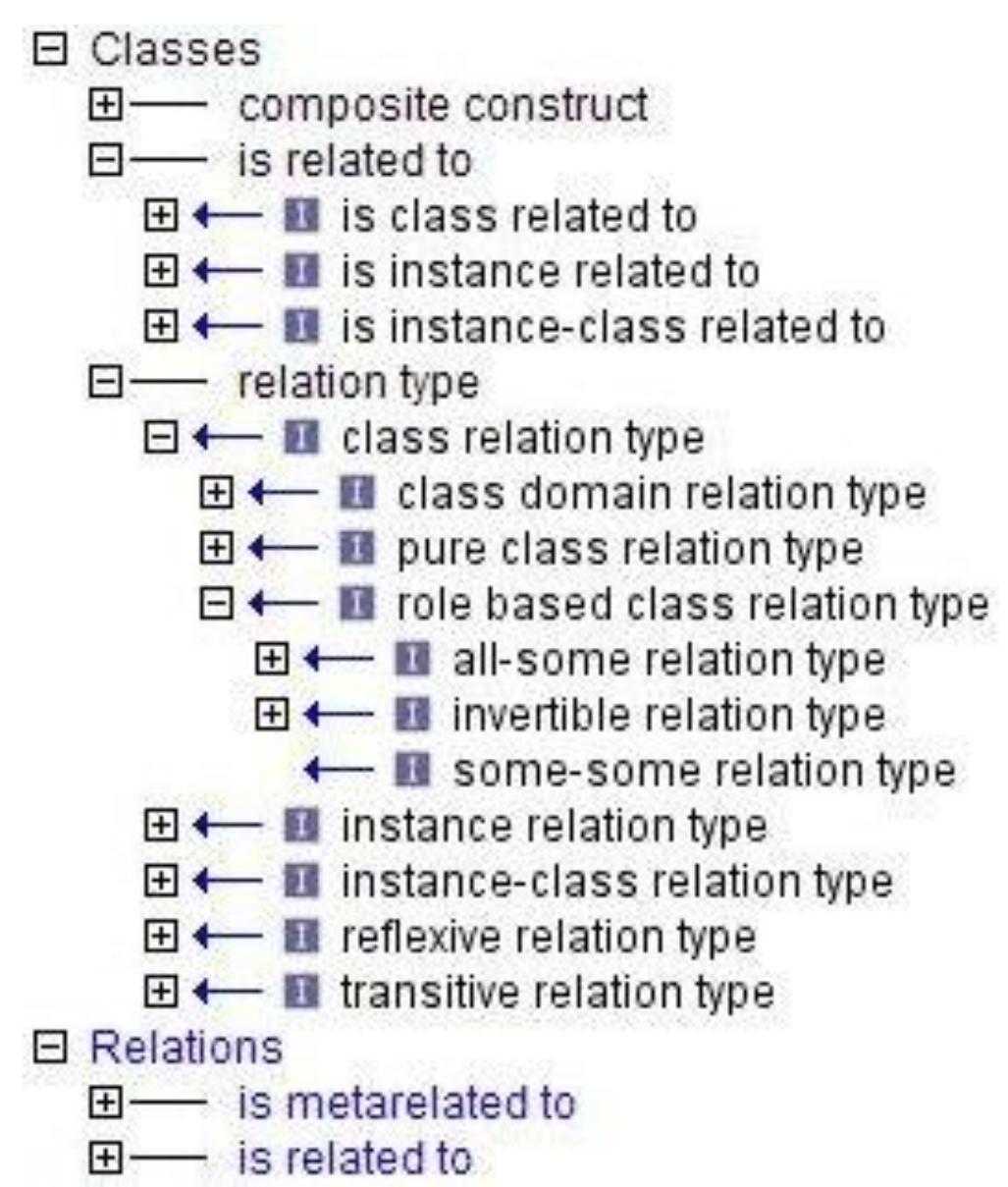




\section{The ease of OBOF in RDF}
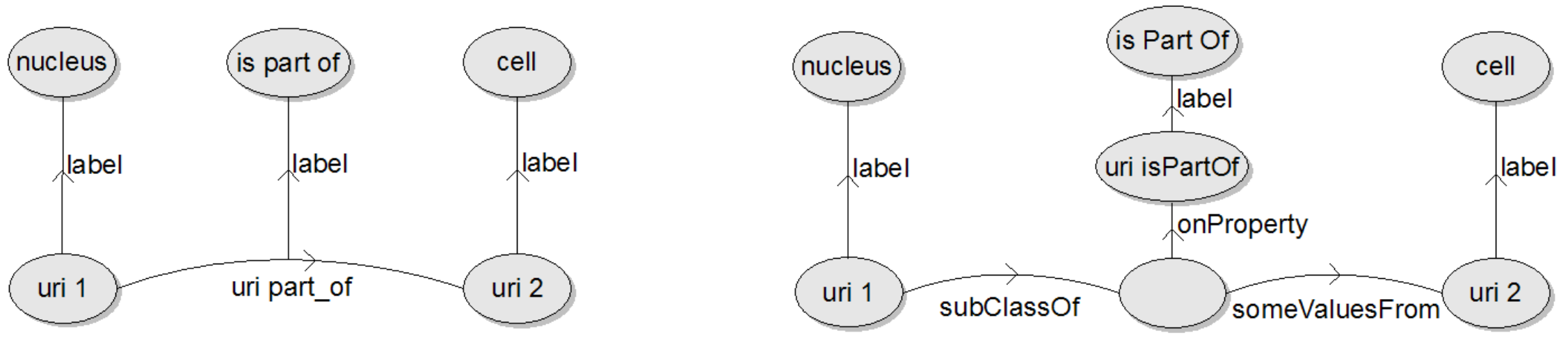

Direct link in OBOF/RDF

Indirect link in OWL/RDF

OBOF provides an all-some semantics for relations between classes, modeled with a single arrow. 


\section{METAREL integration with SPARUL}

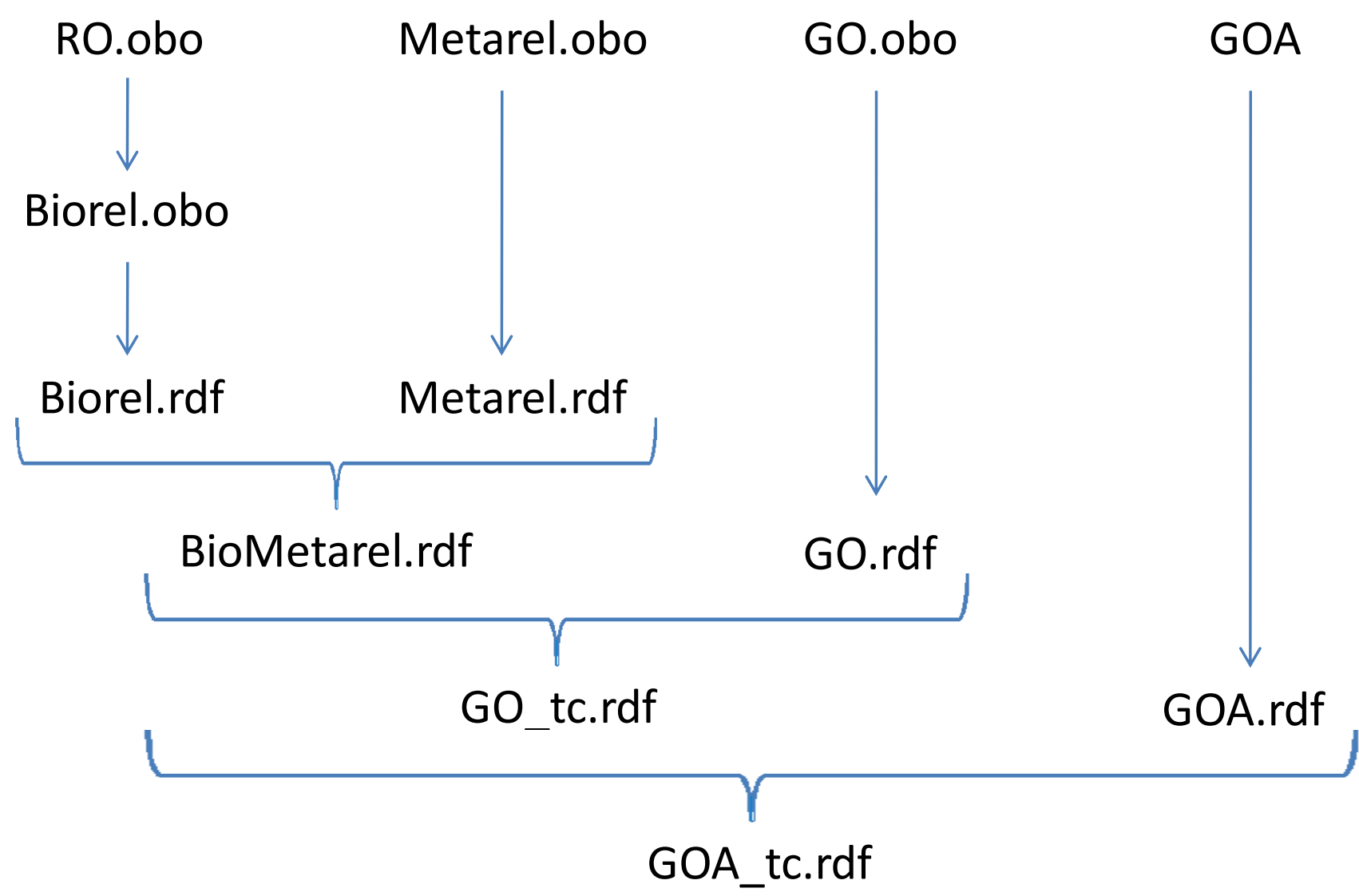

_tc: RDF graphs with total relational closures 


\section{A SPARUL closure query}

BASE <http://www.semantic-systems-biology.org/>

PREFIX ssb:<http://www.semantic-systems-biology.org/SSB\#>

INSERT INTO GRAPH $<25$. H_sapiens_tc $>$ \{

?class1 ?resulting_relation ?class3.

\}

WHERE \{

GRAPH $<25$.H_sapiens_tc $>\{$

?class1 ?first_relation ?class2.

\}

GRAPH <gene_ontology_edit_tc $>\{$

?class2 ?second_relation ?class3.

\}

GRAPH <biometarel> \{

?composite ssb:first_relation ?first_relation.

?composite ssb:second_relation ?second_relation.

?composite ssb:resulting_relation ?resulting_relation.

\}

With 'this query, any pattern of composite

relations is automatically retrieved from

BioMetarel and updated over GO Annotations. 


\section{Next section}

1. Introduction

2. Approach and architecture

3. Relational closures

4. Results

5. Conclusions 


\section{Problem statement}

\section{Proteins:}

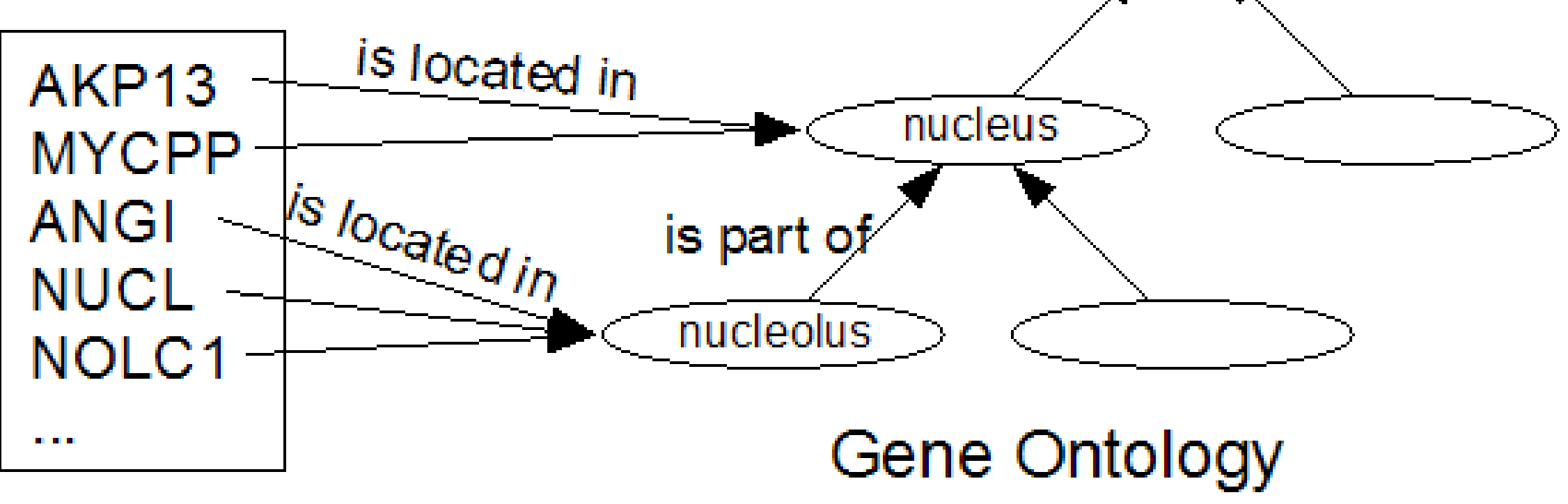

We want to get all the proteins that are located in the nucleus (with an easy SPARQL query). 


\section{Relational closures}

- Reflexivity:

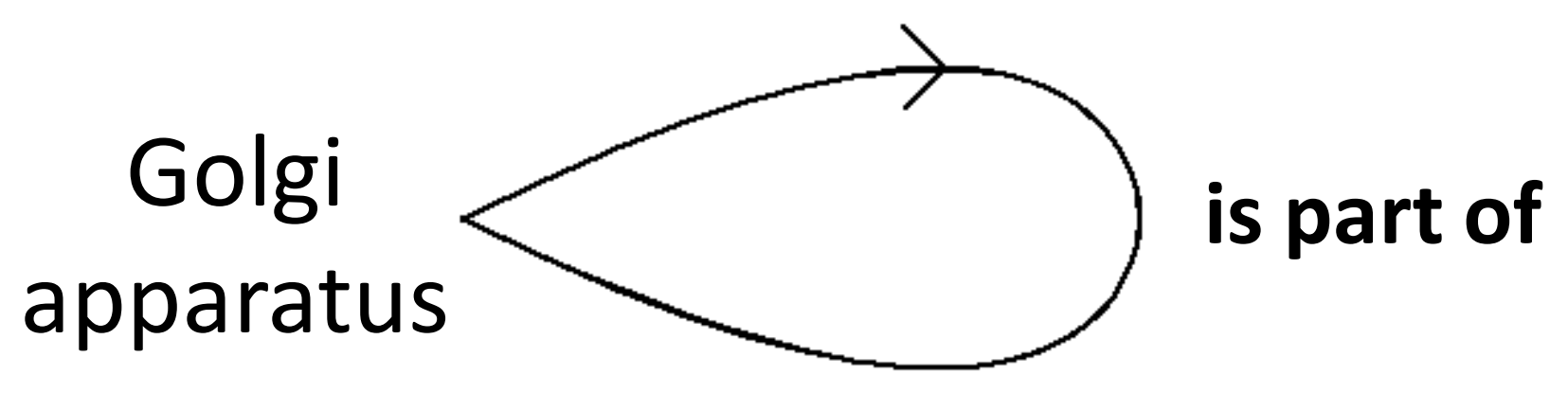




\section{Relational closures}

- Transitivity:

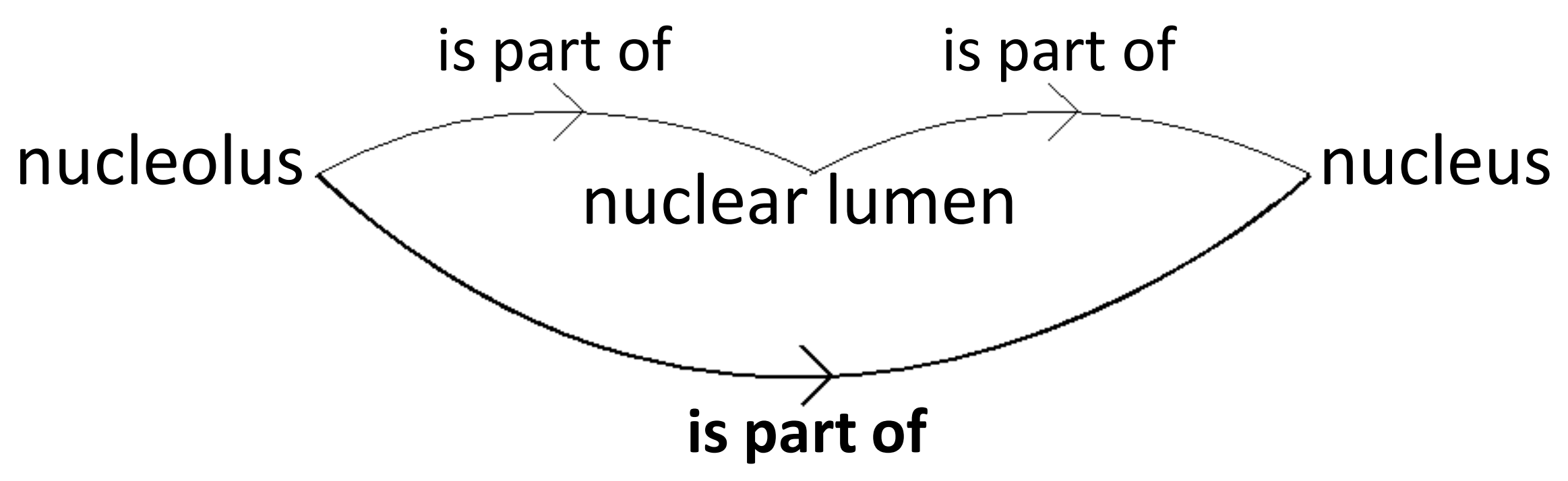




\section{Relational closures}

- Priority over is_a:

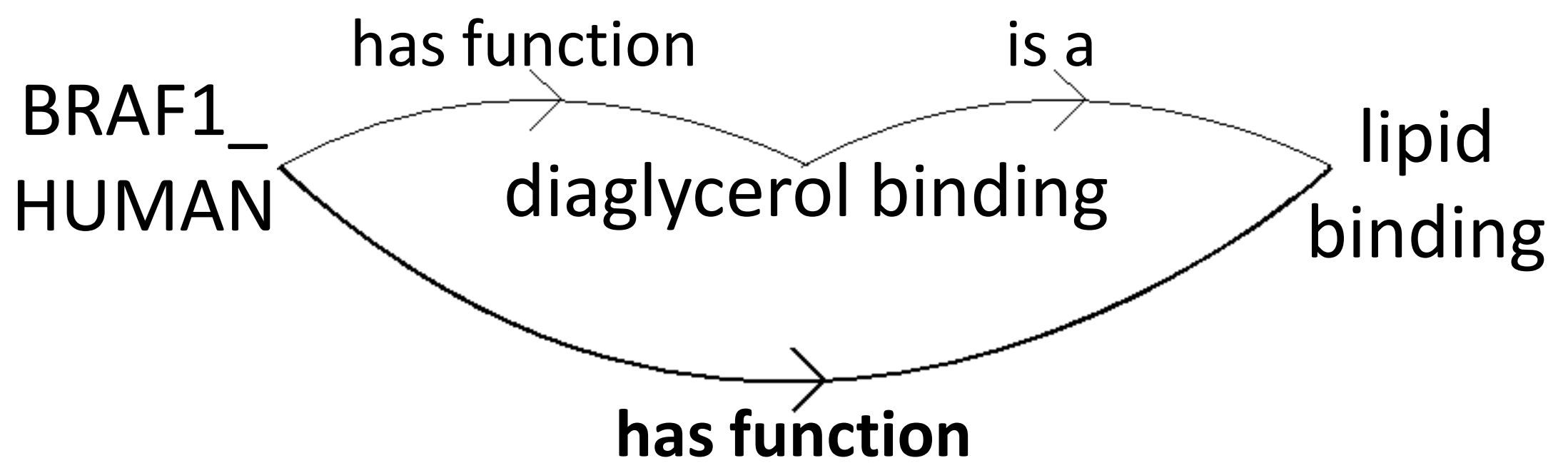




\section{Relational closures}

- Superrelations:

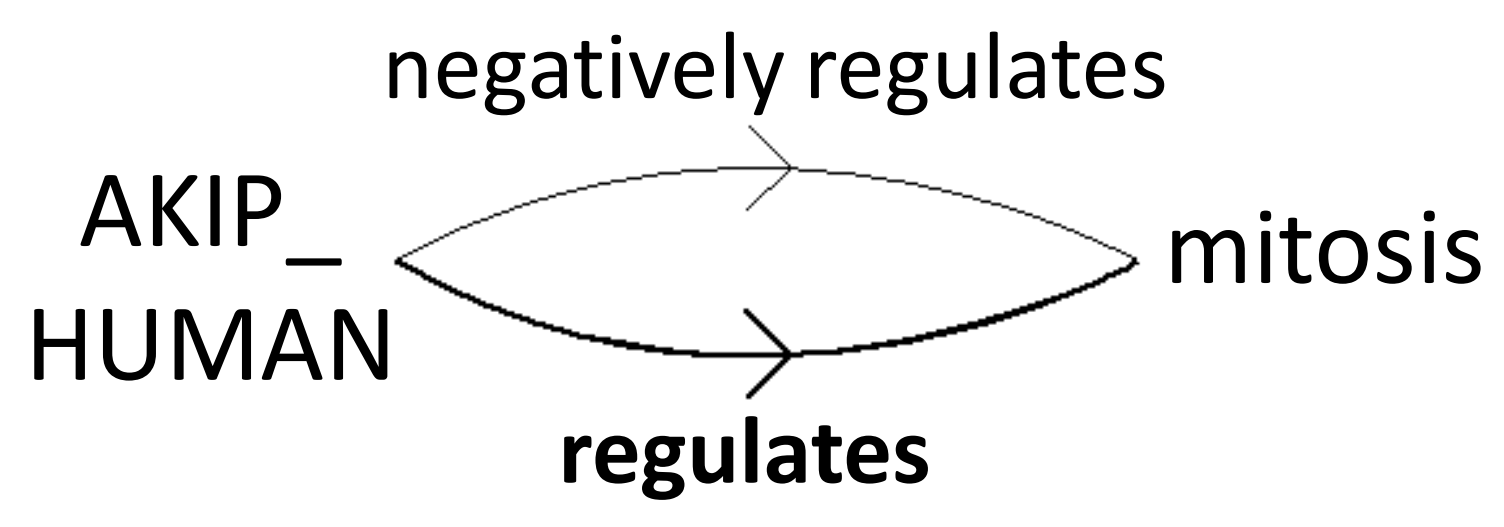




\section{Relational closures}

- Compositions:

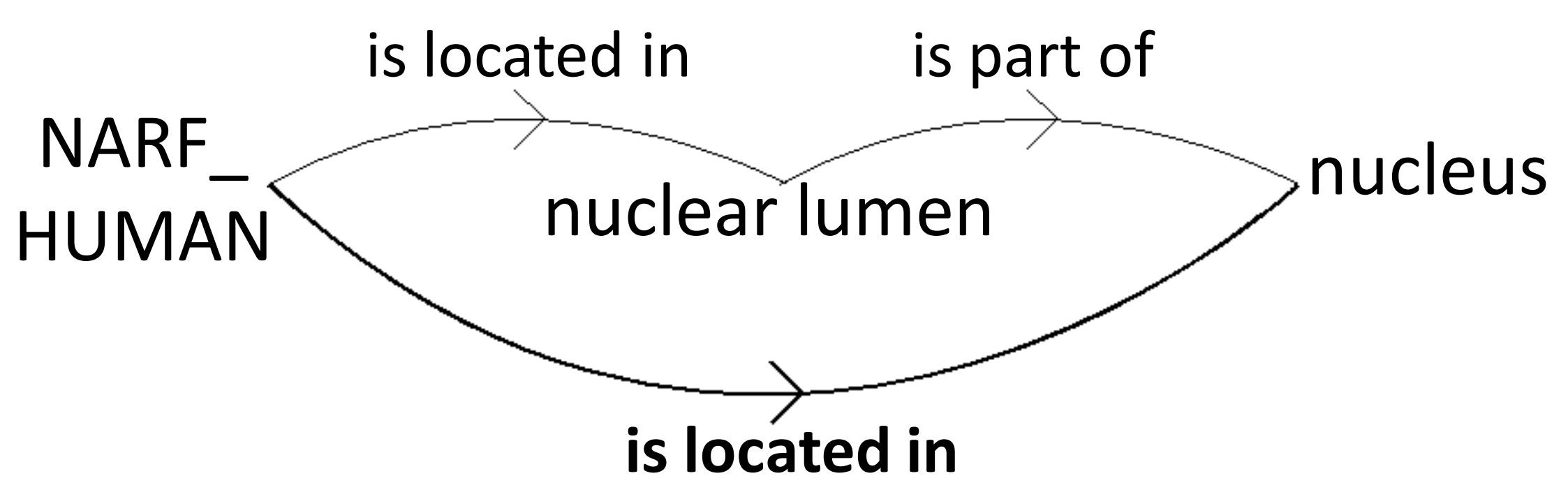




\section{Relational closures}

- Inverses? No!

is part of

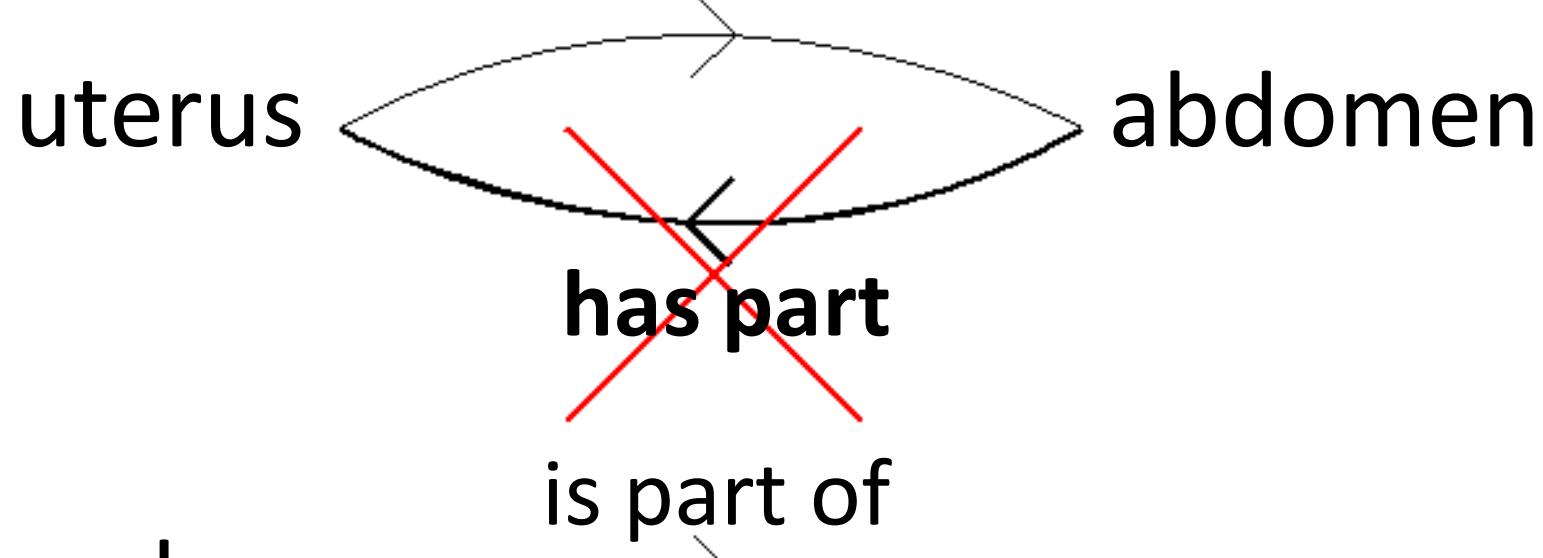

OK: nuclear

lumen

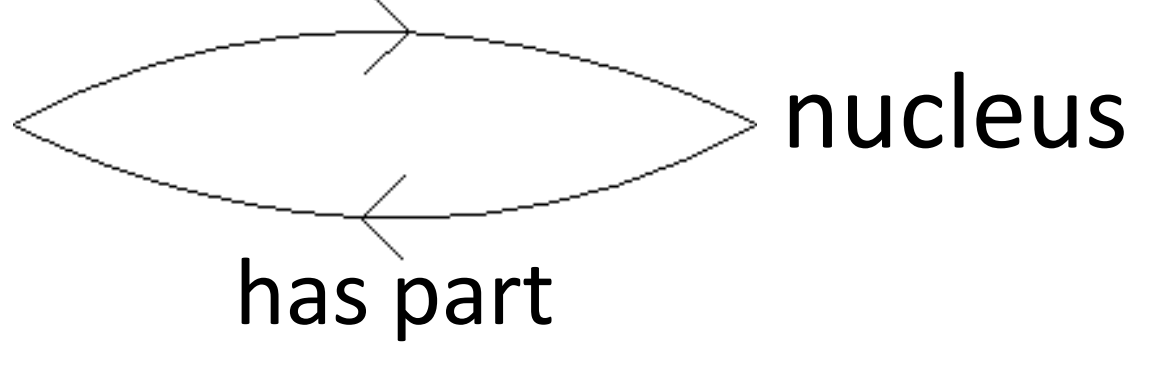

OBO ontologies need inverse relations where automated systems cannot infer them! 


\section{Next section}

1. Introduction

2. Approach and architecture

3. Relational closures

4. Results

5. Conclusions 


\section{Results}

- Millions of new relations were inferred:

GO: from $0.57 \mathrm{~m}$ triples to $1.2 \mathrm{~m}$ triples

GOA (human): from $3.3 \mathrm{~m}$ to $4.0 \mathrm{~m}$ triples

GOA (all): about 200 million triples

- SPARQL queries on the closure graphs give all the expected results in a short time

- Complex queries can easily be addressed in this architecture 


\section{A complex query}

Which proteins, in which organisms

- have function ion channel activity

- are located in the lysosome

- participate in ion transport

9 proteins are found for direct annotations

11 proteins are found in closure graphs:

subclasses in the hierarchy are also

considered now 


\section{Simple queries get a simple syntax}

BASE <http://www.semantic-systems-biology.org/> PREFIX rdfs:<http://www.w3.org/2000/01/rdf-schema\#> PREFIX ssb:<http://www.semantic-systems-biology.org/SSB\#> SELECT distinct ?protein

WHERE \{

\{

GRAPH $<25$. H_sapiens_tc $>\{$

?protein ssb:located_in ssb:GO_0005634.

$$
\text { \} }
$$<smiles>C=CC=C</smiles>

Query with total closures for GOA.

The same* query with

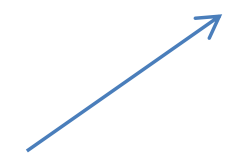
only transitive closures in GO. $\left({ }^{*}\right.$ But didn't we forget something?)

BASE <http://www.semantic-systems-biology.org/>

PREFIX rdfs:<http://www.w3.org/2000/01/rdf-schema\#> PREFIX ssb:<http://www.semantic-systems-biology.org/SSB\#> SELECT distinct ?protein WHERE \{

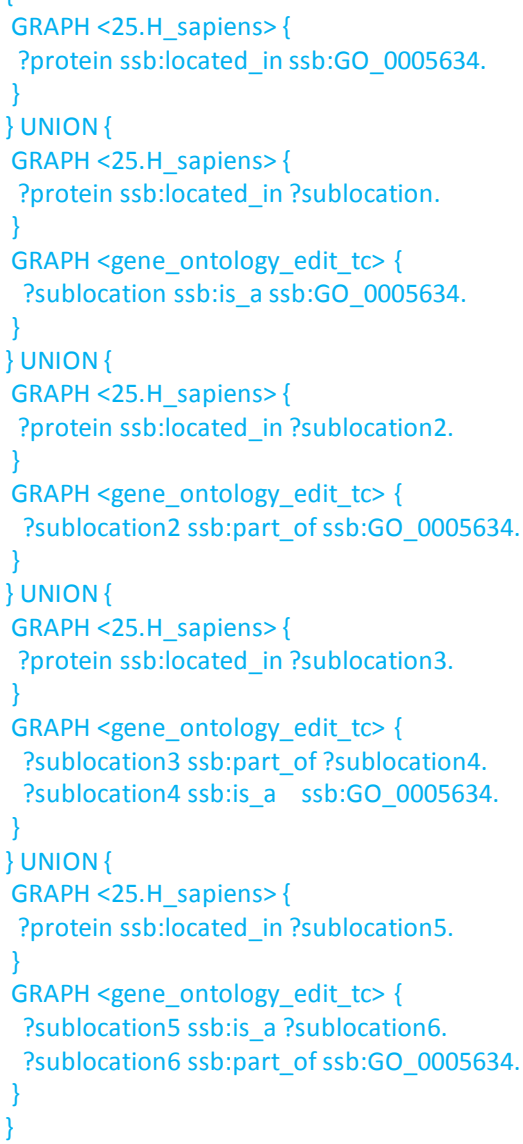




\section{Last section}

1. Introduction

2. Approach and architecture

3. Relational closures

4. Results

5. Conclusions 


\section{Conclusions}

- RDF-based ontologies benefit from preinferenced relational closures for performant SPARQL querying

- SPARUL can infer millions of RDF triples with a small set of closure queries

- Metarel can be used to initiate such closures by providing all the necessary semantics for relations 


\section{Acknowledgements}

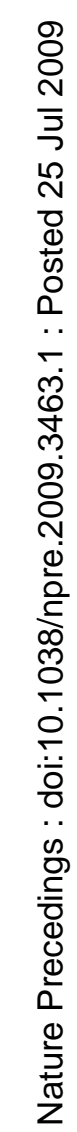

- Erick Antezana (UGent, BE)

- Nirmala Seethappan (NTNU, NO)

- Bjørn Lindi (NTNU, NO)

- Bernard De Baets (UGent, BE)

- Vladimir Mironov (NTNU, NO)

- Martin Kuiper (NTNU, NO)

http://www.semantic-systems-biology.org/metarel

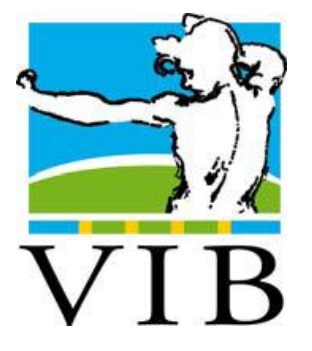

$\mathrm{NT} \mathrm{N} \mathrm{U}$

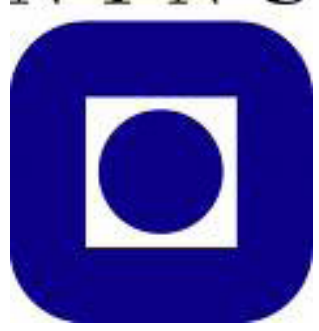


